\title{
Giant dural arteriovenous fistula in an infant
}

\author{
Charles Peper ${ }^{1}$, Joe Iwanaga ${ }^{1,2}$, Aaron S. Dumont ${ }^{1}$, R. Shane Tubbs ${ }^{1,2,3,4,5,6}$ \\ ${ }^{1}$ Department of Neurosurgery, Tulane Center for Clinical Neurosciences, Tulane University School of Medicine, New Orleans, LA, ${ }^{2}$ Department of \\ Neurology, Tulane Center for Clinical Neurosciences, Tulane University School of Medicine, New Orleans, LA, ${ }^{3}$ Department of Structural \& Cellular \\ Biology, Tulane University School of Medicine, New Orleans, LA, ${ }^{4}$ Department of Neurosurgery and Ochsner Neuroscience Institute, Ochsner Health \\ System, New Orleans, LA, USA, ${ }^{5}$ Department of Anatomical Sciences, St. George's University, St. George's, Grenada, ${ }^{6}$ Department of Surgery, Tulane \\ University School of Medicine, New Orleans, LA, USA
}

Abstract: Dural arteriovenous fistulas (dAVFs) are commonly encountered by the neurosurgeon. Herein, we present a case illustration of an infant presenting with an extremely large fistula that took up a significant part of the intracranial volume. A one-month-old female presented with irritability and failure to thrive. She was the product of a 35-week pregnancy and was delivered vaginally without complications or a difficult labor. Based on the findings of magnetic resonance imaging, the diagnosis of a giant dAVF involving the transerve-sigmoid sinuses was made. The patient was scheduled for an arteriogram but died before the procedure could be performed. Such a case illustrates how large some dAVF can become and at a very early age. As in the present case, the patient was minimally symptomatic. Therefore, the time to intervention after diagnosis is thus, sometimes, critical.

Key words: Dural arteriovenous fistula, Infant, Dural venous sinus

Received April 27, 2021; 1st Revised July 10, 2021; 2nd Revised July 14, 2021; Accepted July 21, 2021

\section{Introduction}

A dural arteriovenous fistula (dAVF) occurs when at least one meningeal artery connects directly to either a dural venous sinus or cortical (leptomeningeal) vein. This event results from angiogenesis following head trauma, surgery, thrombosis, infection, or as a spontaneous occurrence. In any case, vascular endothelial growth factor is found in high concentrations near the dAVF [1].

Commonly in dAVFs involving the transverse-sigmoid sinus [2], oxygenated blood that flows directly into a dural sinus causes the vessel to arterialize and form a fistulous pouch, which can occur both at the site of, or distal to the site of the shunt or shunts. Ectasia can also occur in the corti-

\footnotetext{
Corresponding author:

Joe Iwanaga (iD

Department of Neurosurgery, Tulane Center for Clinical Neurosciences, Tulane University School of Medicine, New Orleans, LA 70112, USA E-mail: iwanagajoeca@gmail.com
}

cal veins proximal to the shunt location in Cognard type IV dAVFs [3].

dAVFs are routinely categorize based on shunt location, source of aberrant arterial supply, and venous drainage pattern [3, 4]. Although no classification system for fistula pouch size has yet been proposed, clinicians observe wide variations. Herein, we present a case-study of a giant dAVF fistula in an infant involving the transverse-sigmoid sinus. Such a case illustrates how large some dAVF can become and at a very early age. As in the present case, the patient was minimally symptomatic. Therefore, the time to intervention after diagnosis is thus, sometimes, critical.

\section{Case Report}

A one-month-old female presented with irritability and failure to thrive. She was the product of a 35-week pregnancy and was delivered vaginally without complications or a difficult labor. Prenatal ultrasonography was not performed. Her Apgar score was 7. The parents reported failure to thrive and an infant who was minimally responsive although she would 
open her eyes and was able to follow objects. Her pupils were more or less symmetrical. Blood chemistry panels were within normal limits. On physical exam, the infant moved all extremities to stimulation and grimaced to pinch. The anterior fontanelle was full and tense and the head circumference was $42 \mathrm{~cm}$. The sutures of the calvaria were split. There was an upgaze palsy. Reflexes were found to be hypotonic. No cutaneous stigmata were identified.

A subsequent magnetic resonance imaging (MRI) identified a large, somewhat spherical mass with significant flow void filling most of the posterior cranial fossa and extending superiorly to the underside of the calvaria (Fig. 1A-D). In total, the mass filled approximately one half of the intracranial volume. No cerebellum was identified and the brainstem was significantly compressed against the clivus (Fig. 1B). Hydrocephalus was present, especially involving the temporal horns (Fig. 1A, C, D). The internal jugular veins were dilated (Fig. 1E) and the circle of Willis and vertebrobasilar vessels appeared normal (Fig. 1F) although the resolution of the imaging was poor primarily due to the age of the patient, therefore, based on the findings of MRI, the diagnosis of a giant dAVF involving the transverse-sigmoid sinuses was made. The patient was scheduled for an arteriogram but died before the procedure could be performed.

\section{Discussion}

Because of the ability for dAVFs to recruit collateral arterial supply, proximal embolization is likely to show resolution of the fistulous connection on immediate postoperative angiography, but durability of these cures may be overestimated [5]. Therefore, in non-surgical treatment, transvenous embolization of the fistula pouch in the dural sinus remains the best deterrent for recurrence of shunting $[2,6]$. Transarterial balloon-assisted embolization with transvenous balloon protection also provides a high rate of clinical cure or remission even at follow-up angiography [2, 7]. In a giant dAVF, such as the one presented here, collateral arterial supply builds a robust framework of feeding vessels [8, 9].

When embolization for dAVFS appears inappropriate or insufficient, surgery and radiosurgery are considered. Several published reviews suggest the effectiveness of a multidisciplinary approaches to large or difficult dAVFs. Friedman et al. [10] recommended a staged endovascular and radiosurgery for patient's presenting with low risk dAVFs of the transverse and sigmoid sinues after no intracranial hemorrhage or non-hemorrhagic neurological disorder was documented after a mean follow up time of 50 months in patients who had received radiosurgery, 20 of whom (87\%) also
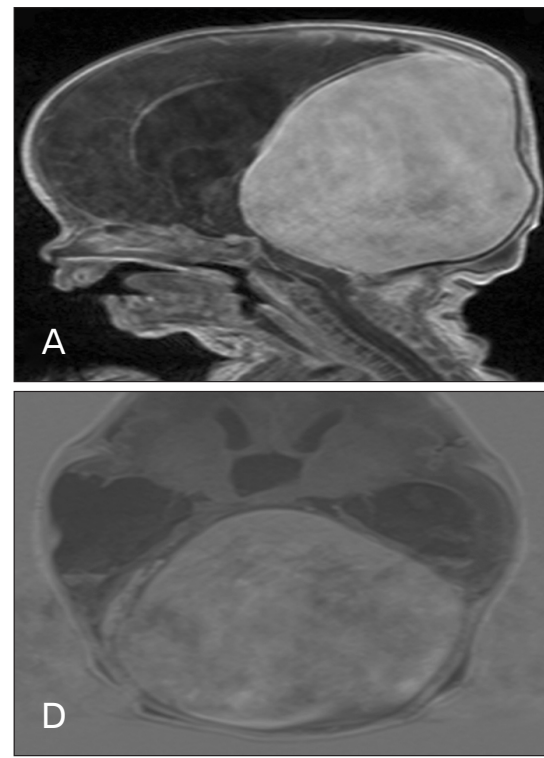
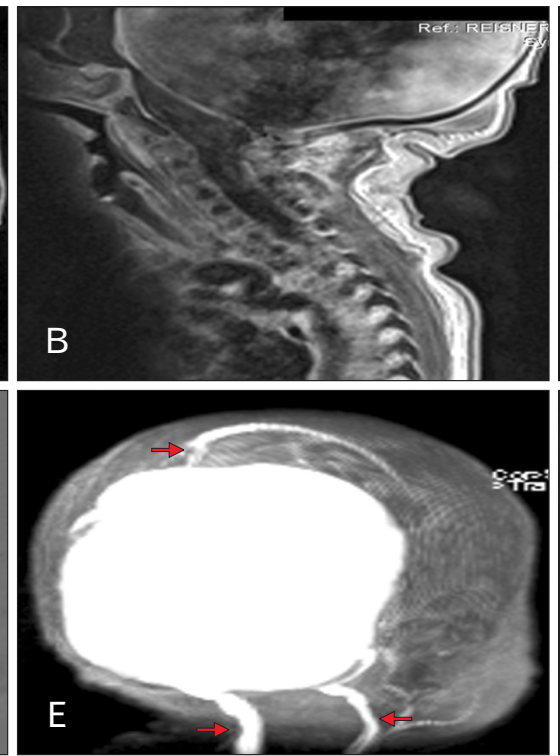
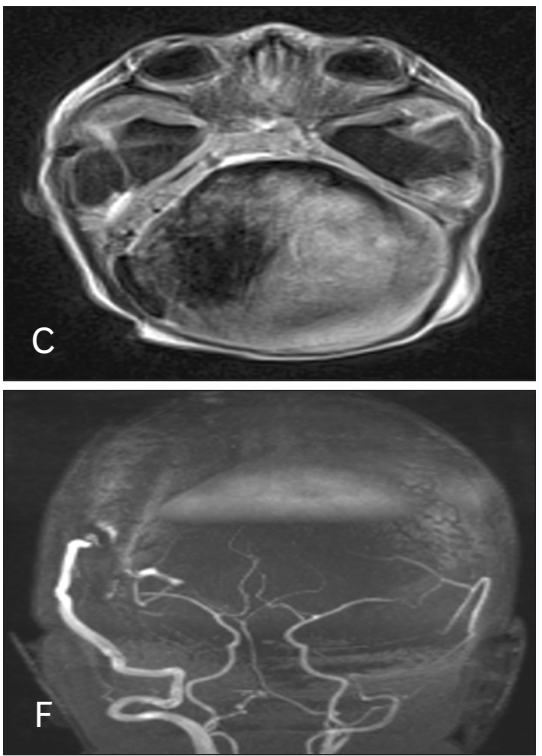

Fig. 1. (A) T1-weighted magnetic resonance imaging (MRI) noting the large intracranial mass. Hydrocephalus is evident in the lateral ventricles. (B) T1-weighted MRI noting the compression of the contents of the posterior cranial fossa. (C) T1-weighted MRI noting the large intracranial mass filling most of the posterior cranial fossa. Hydrocephalus is evident in the temporal horns. (D) Axial image noting the dilated ventricular system. Hydrocephalus is evident in the third ventricle. (E) Scout image illustrating the giant mass and dilated internal jugular veins (lower arrows). Note the superior sagittal sinus at the upper arrow. (F) Note the normal appearance of the components of the circle of Willis and vertebrobasilar system. 
received transarterial embolization.

In another case study of a large intracranial dAVF, a multidisciplinary approach required embolization, surgical occlusion, and radiosurgery for successful treatment of the lesion which involved the sagittal, straight, and bilateral transverse sinuses [8]. This was a 21-year-old patient with headache, visual loss, and pulsatile tinnitus. An occipital bruit was identified on physical swelling along with optic nerve edema. On imaging, a large dAVF was found fed by the middle meningeal and meningohypophsial vessels with fistulous involvement of the transverse, sigmoid, and straight sinuses. It was noted that embolization alone would have been unable to obliterate all small feeding vessels, although it was still useful in occluding five large feeding vessels. Surgical skeletonization of the venous sinuses and ligation of the remaining feeding vessels was then performed, followed by radiosurgery to all sites of fistulous connection. At one-year follow-up, a repeat angiogram found complete resolution of the dAVF. Such a case illustrates that a combination of radiosurgery and open surgical ligation can be successful in dealing with larger dAVFs. However, due to the huge size of the dAVF in our case, both methods would have unlikely reversed the patient's clinical outcome. Additionally, such a case illustrates how large some dAVF can become and a very early age. As in the present case, the patient was minimally symptomatic. Therefore, the time to intervention after diagnosis is thus, sometimes, critical. Although the exact embryological processes involved in dAVF are not fully understood, due to the large size of our patient's lesion, the fistula is assumed to have developed inutero. This is most likely to occur during 40 to $80 \mathrm{~mm}$ when the dural venous sinsues are formed [11]. Additionally, although most dAVFs develop in adults, cases of child and even fetal development have been reported [12].

In conclusions, our case illustrates the wide variation seen in size of venous distension in dAVFs. When taken in consideration with the clinical importance of obliterating the entire fistula pouch in successful and durable treatment of dAVFs, this may suggest the possibility for development of a classification system of dAVFs based on the extent of distension of the fistula.

\section{ORCID}

Charles Peper: https://orcid.org/0000-0002-3520-4359

Joe Iwanaga: https://orcid.org/0000-0002-8502-7952
Aaron S. Dumont: https://orcid.org/0000-0002-8077-8992

R. Shane Tubbs: https://orcid.org/0000-0003-1317-1047

\section{Author Contributions}

Conceptualization: JI, RST. Data acquisition: RST. Data analysis or interpretation: CP, ASD. Drafting of the manuscript: CP, JI. Critical revision of the manuscript: ASD, RST. Approval of the final version of the manuscript: all authors.

\section{Conflicts of Interest}

No potential conflict of interest relevant to this article was reported.

\section{References}

1. Li Q, Zhang Q, Huang QH, Fang YB, Zhang ZL, Xu Y, Liu JM. A pivotal role of the vascular endothelial growth factor signaling pathway in the formation of venous hypertension-induced dural arteriovenous fistulas. Mol Med Rep 2014;9:1551-8.

2. Xu K, Yang X, Li C, Yu J. Current status of endovascular treatment for dural arteriovenous fistula of the transverse-sigmoid sinus: a literature review. Int J Med Sci 2018;15:1600-10.

3. Cognard C, Gobin YP, Pierot L, Bailly AL, Houdart E, Casasco A, Chiras J, Merland JJ. Cerebral dural arteriovenous fistulas: clinical and angiographic correlation with a revised classification of venous drainage. Radiology 1995;194:671-80.

4. Borden JA, Wu JK, Shucart WA. A proposed classification for spinal and cranial dural arteriovenous fistulous malformations and implications for treatment. J Neurosurg 1995;82:166-79.

5. Adamczyk P, Amar AP, Mack WJ, Larsen DW. Recurrence of "cured" dural arteriovenous fistulas after Onyx embolization. Neurosurg Focus 2012;32:E12.

6. Dawson RC 3rd, Joseph GJ, Owens DS, Barrow DL. Transvenous embolization as the primary therapy for arteriovenous fistulas of the lateral and sigmoid sinuses. AJNR Am J Neuroradiol 1998;19:571-6.

7. Piechowiak E, Zibold F, Dobrocky T, Mosimann PJ, Bervini D, Raabe A, Gralla J, Mordasini P. Endovascular treatment of dural arteriovenous fistulas of the transverse and sigmoid sinuses using transarterial balloon-assisted embolization combined with transvenous balloon protection of the venous sinus. AJNR Am J Neuroradiol 2017;38:1984-9.

8. Giller CA, Barnett DW, Thacker IC, Hise JH, Berger BD. Multidisciplinary treatment of a large cerebral dural arteriovenous fistula using embolization, surgery, and radiosurgery. Proc (Bayl Univ Med Cent) 2008;21:255-7.

9. Reynolds MR, Lanzino G, Zipfel GJ. Intracranial dural arteriovenous fistulae. Stroke 2017;48:1424-31.

10. Friedman JA, Pollock BE, Nichols DA, Gorman DA, Foote RL, 
Stafford SL. Results of combined stereotactic radiosurgery and transarterial embolization for dural arteriovenous fistulas of the transverse and sigmoid sinuses. J Neurosurg 2001;94:88691.

11. Mullan S, Mojtahedi S, Johnson DL, Macdonald RL. Embryo- logical basis of some aspects of cerebral vascular fistulas and malformations. J Neurosurg 1996;85:1-8.

12. Kaushik KS, Acharya UV, Ananthasivan R, Girishekar B, Reddy P. Fetal dural sinus malformation. Neurology 2020;95:452-3. 